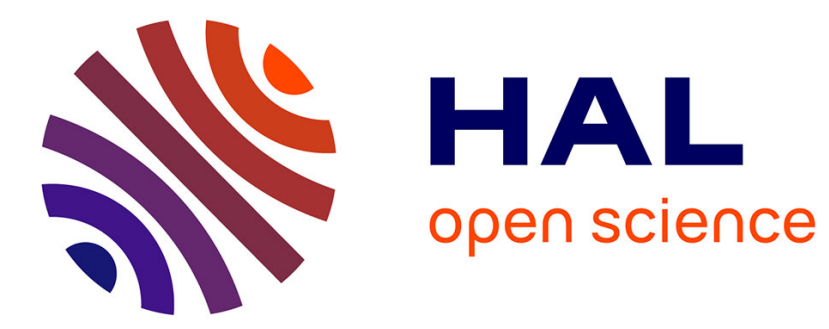

\title{
Effect of Phase Noise on the Frequency Calibration of a Tunable Laser by Heterodyne Signal Filtering
}

Wenhui Yu, Pierre Pfeiffer, Sylvain Lecler, Joel Fontaine

\section{To cite this version:}

Wenhui Yu, Pierre Pfeiffer, Sylvain Lecler, Joel Fontaine. Effect of Phase Noise on the Frequency Calibration of a Tunable Laser by Heterodyne Signal Filtering. IEEE Journal of Quantum Electronics, 2018, 54 (6), pp.1-8. 10.1109/JQE.2018.2879482 . hal-02379926

\section{HAL Id: hal-02379926 https://hal.science/hal-02379926}

Submitted on 25 Nov 2019

HAL is a multi-disciplinary open access archive for the deposit and dissemination of scientific research documents, whether they are published or not. The documents may come from teaching and research institutions in France or abroad, or from public or private research centers.
L'archive ouverte pluridisciplinaire HAL, est destinée au dépôt et à la diffusion de documents scientifiques de niveau recherche, publiés ou non, émanant des établissements d'enseignement et de recherche français ou étrangers, des laboratoires publics ou privés. 


\title{
Effect of Phase Noise on the Frequency Calibration of a Tunable Laser by Heterodyne Signal Filtering
}

\author{
Wenhui Yu, Pierre Pfeiffer, Sylvain Lecler and Joël Fontaine
}

\begin{abstract}
Using a frequency comb as frequency reference to calibrate the instantaneous frequency of a tuning laser allows high spectral resolution and a wide calibration range. A classical method to obtain the instantaneous frequency of the laser under test is by filtering the heterodyne signal between the frequency comb and the tunable laser with a narrow bandpass filter. For free-running femtosecond lasers, the phase noise of the comb lines affects the instantaneous frequency of the heterodyne signal and the envelope of the filtered calibration signal. In this paper, the characteristics of the frequency calibration signal envelope is analyzed by modeling. Three different filters are used to consider the envelope characteristics. Simulation results show that the probability density function (PDF) of the envelope amplitude tend to be a uniform distribution at higher phase noise level. At low tuning speed, the PDF distributions are the same at symmetric frequency positions of the passband of the filter. At high tuning speed, their distributions become different. The standard deviation of the center of mass becomes larger at higher phase noise level and higher tuning speed.
\end{abstract}

Index Terms-filter, heterodyne, optical frequency comb, phase noise.

\section{INTRODUCTION}

$\mathbf{M}$ EASURING the dynamic frequency of a laser is important in revealing the working mechanism of the laser and also for applications where frequency modulation is required. For example, in light detection and ranging ( $\mathrm{Li}-$ DAR) systems based on frequency modulated continuous wave (FMCW), the instantaneous optical frequency of the swept laser should be monitored for passive linearization of the tuning speed [1], [2]. In tunable laser spectroscopy, the frequency should be calibrated in order to record the spectral features correctly [3], [4], [5]. Conventional spectrometers such as Fabry-Perot etalon and dispersion gratings, have difficulties in fulfilling both high spectral resolution and wide measuring range. Recently, spectral dynamics analysis based on optical heterodyne method has shown great power in offering high spectral resolution [6], [7]. Meanwhile, in these heterodyning spectrometers, the frequency combs have been used as the local oscillators (LOs) to heterodyne with the laser under test (LUT) [3], [4], [8], allowing for wideband and high precision frequency calibration.

To analyze the spectral dynamics of the LUT, the frequency is down-converted by the LO laser into the radio frequency (RF) region and thus can be analyzed electronically. The dynamic frequency of the LUT is obtained by processing the heterodyne signal, which in general, can be categorized into two types of processing methods. The first is the spatiotemporal

The authors are with the ICube-IPP, University of Strasbourg, CNRS, 67412 Illkirch, Strasbourg (Email: wenhui.yu@unistra.fr). presentation of the dynamic frequency, forming a spectrogram by offline signal processing, such as the short time Fourier transform, or wavelet transform. These methods can provide the spectral components of the obtained heterodyne signal within the analyzing time windows, forming the spectrogram of the dynamic signal. However, it requires offline signal processing, thus the heterodyne signal should be digitized with a sampling speed greater than the Nyquist frequency and the digitized data is then recorded for processing. The bandwidth and the registered memory depth of the data acquisition system limit the maximum frequency that can be recorded. The second method uses frequency discriminators based on narrowband bandpass filters (NBFs). The frequency difference is converted to the difference of the intensity of the output filtered signal. The later method is online real-time filtering and is particularly suitable in some situations, for example, the filtered signal can be used as the triggering signal for sampling another signal.

In heterodyne detection, the phase of the IF (intermediate frequency) signal is the phase difference between the electric fields of the LUT and the LO. The spectrum of the RF signal is the exact down-converted version of the optical spectrum of the LUT only when the contribution of the phase noise of the LO is neglectable. Thus, highly stable and narrow linewidth LO lasers are required. However, for free-running (that is without phase-lock-loop for frequency stabilization) singlefrequency solid-state lasers, the typical measured linewidth is a few kilohertz over one second measuring time and it is often in the megahertz rangefor monolithic diode lasers. Even though sub hertz linewidth of laser diodes has been achieved by stabilizing based on high-finesse cavities and phase locking between lasers [8], the phase locking process is not a trivial task. This is also true for completely locking the frequency combs. To avoid the complexity of phase locking of a frequency comb, free-running combs are sometimes used and the phase noise is considered as a systematic noise contributor [1], [9]. On the other hand, the weak power of the individual comb line results in a relatively low signal-to-noise ratio, which in turn, results a significant corruption of the amplitude of the beat signal. A knowledge of the effect of phase noise on the amplitude helps us to determine what is the origin of the amplitude noise and further increases the signal-to-noise ratio correspondingly.

In this paper, the effect of phase noise of the LO laser on the spectral resolution using a heterodyne with filtering method is considered. To achieve this, an ideal linearly tuned laser is considered as the dynamic frequency source. In practice a real tunable laser can only be tuned quasi-linearly. This assumption does not lose its generality, since within the small 
passband of the NBF, the chirping characteristic of the tuning laser can be well approximated by its first order approximation and the whole dynamic process is composed by sections having different chirp rate [10]. In the following sections, we will discuss how the phase noise of the LO makes the envelope of the calibration signal deviate from the amplitudefrequency response curve of the NBF. As a result, random oscillations in the envelope occurs [3]. To characterize the random oscillation, the probability density function (PDF) of the envelope amplitude at different frequency positions of the calibration signal is computed. In the simulation, we started from the output signal of the NBF in a truncated time interval, where the heterodyne signal is simulated by a linear chirped signal that is contaminated by a Gaussian phase noise.

This paper is organized as follows: in Section 2, we first describe a typical tuning frequency calibration system using frequency comb and filtering approach. It should be noted that the result can also be applied to the single frequency LO laser. In Section 3, the model for analyzing the phase noise effect is built. Then in Section 4, simulation results are presented and discussed.

\section{TUNING FREQUENCY CALIBRATION USING HETERODYNING AND FILTERING}

\section{A. System description}

A typical setup of frequency calibration of a tunable laser using heterodyning and a filtering method is shown in Fig. 1(a), similar to the setup described in [3], [4]. An optical frequency comb is used as the LO. The optical frequency comb is generated from a mode-locked femtosecond laser, that can be regarded as formed by a large number of single frequency lasers whose frequencies are separated by the pulse repetition rate. This structure of spectrum allows for a wide range frequency calibration of the tunable laser .

The beams from the tunable laser and the frequency comb interfere on the photodetector. In general, the photocurrent contains the frequency components generated by heterodyning between the different comb lines as well as the frequency components generated by heterodyning between the tuning LUT and the comb lines. The former includes the repetition rate of the comb $\left(f_{r}\right)$ and its harmonies whereas the latter includes the beat signal frequencies (between the LUT and the nearest comb line) and its offset versions by $n f_{r}$. By setting the passband of the NBF away from the repetition rate $f_{r}$, the high power of $f_{r}$ and its harmonies are filtered out. Thus, only the heterodyne signal between the LUT and the nearest comb line should be considered, which can be written in complex form (consider only the AC term):

$$
\tilde{S}_{i}(t)=2 R \sqrt{P_{T} P_{n}} \exp \left[i 2 \pi\left(\nu_{T}-\nu_{n}\right) t+i\left(\theta_{T}-\theta_{n}\right)\right]
$$

Here, $R$ is the responsivity of the photodetector. $P_{T}$ and $P_{n}$ are the optical powers, $\nu_{T}$ and $\nu_{n}$ are the optical frequencies and $\theta_{T}$ and $\theta_{n}$ are the phase noises of the tunable laser and the comb line respectively. After the narrow bandpass filter, the heterodyne signal passes through only when the frequency is within the passband of the NBF, producing peak signals. Hence, the peak signal are the time markers that
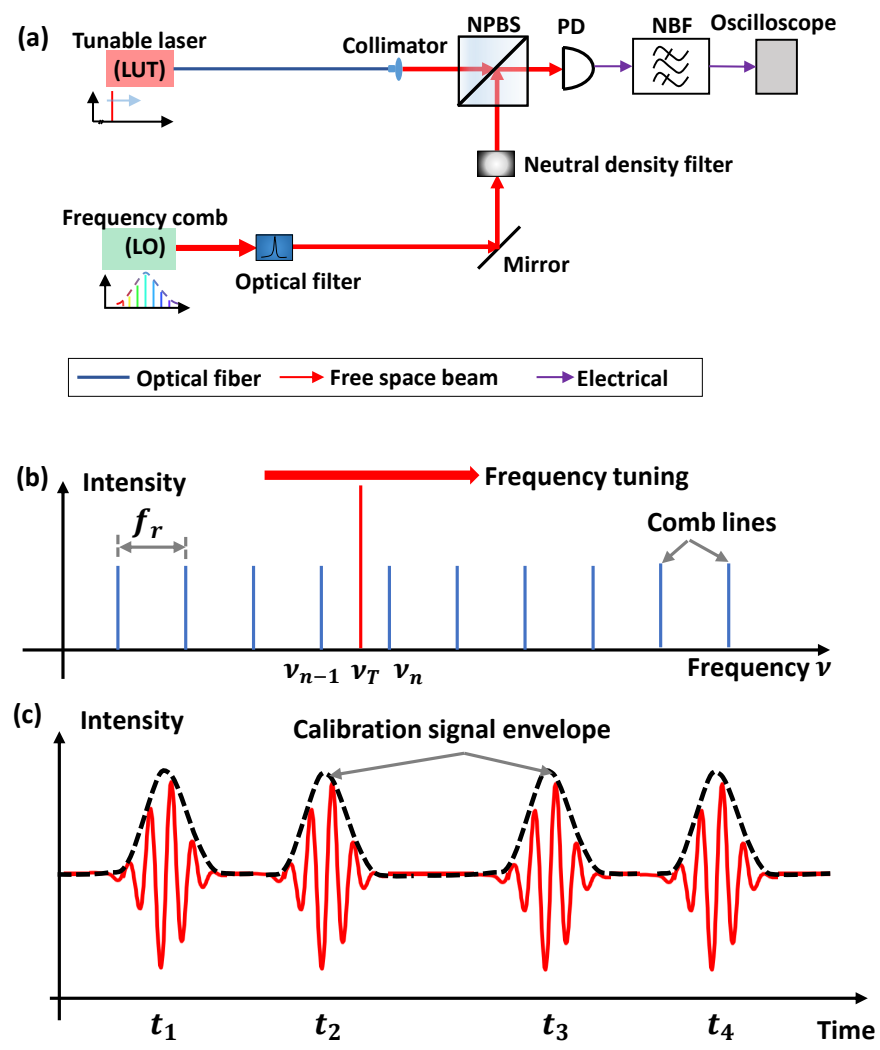

Fig. 1. (a). Schematic of a typical frequency calibration by the filtering heterodyne signal. LUT: laser under test. LO, local oscillator. NPBS: nonpolarized beamsplitter. PD: photodetector. NBF, narrowband filter. (b). Illustration of the frequency position relation between the tunable laser and the frequency comb lines. $f_{r}$ is the repetition rate of the frequency comb. $\nu_{T}$ and $\nu_{n}$ are the frequencies of the tunable laser and its nearest comb line. (c). Illustration of calibration signal. This calibration signal is generated by filtering the heterodyne signal. The peaks correspond to the time when the frequency of the LUT equals $\nu_{n} \pm f_{0}$, where $f_{0}$ is the central frequency of the NBF and $\nu_{n}$ is the optical frequency of the nearest comb line.

correspond to the frequency of the LUT $\nu_{L U T}$ equaling to $\nu_{n} \pm f_{0}$, where $f_{0}$ is the central frequency of the NBF and $\nu_{n}$ is the optical frequency of the nearest comb line. Ideally, the amplitude of the calibration signal envelope follows the amplitude-frequency response curve of the NBF, thus we can determine the instantaneous frequency of the input chirping signal from the envelope of the calibration signal.

\section{B. Distortion of the calibration signal envelope due to phase} noise

The envelope of the calibration signal follows the amplitude-frequency response curve of the NBF only when the rising time of the NBF is smaller than the time required for the tuning heterodyne signal to go through the passband of the NBF. Also, for a phase noise contaminated LO, its phase noise will be transmitted to the heterodyne signal. After filtered by the NBF, the envelope of the calibration signal deviates from the ideal envelope. To determine the position of the centroid of the envelope, the center of mass of the envelope is used, which is defined for a general non-negative function $f(x)$ as: 


$$
x_{c}=\frac{\int_{-\infty}^{+\infty} x f(x) d x}{\int_{-\infty}^{+\infty} f(x) d x}
$$

In what follows, the effect of the phase noise along with the tuning speed on the calibration signal is investigated from analyzing the following two statistics:

(a) the PDF of the envelope amplitude at a given frequency position, and

(b) the distribution of the center of mass under the effect of phase noise

These two statistical quantities are related to the type of phase noise. In the present paper, Gaussian phase noise is analyzed numerically. In the model, the parameters that have effects on the signal filtering process, including the phase noise, the chirp rate of the heterodyne signal and the bandwidth of the NBF, are considered and expressed in normalized form.

\section{MODEL}

In this section, the model for obtaining the PDF of the envelope of the calibration signal is built. For a LUT that is linearly tuned, the heterodyne signal in (1) can be rewritten as a linearly chirped signal:

$$
\tilde{S}_{i}(t)=S_{0} \exp \left[i 2 \pi\left(f_{0}+v t / 2\right) t+i \theta(t)\right]
$$

where $f_{0}$ is the heterodyne frequency at $t=0, v$ is the frequency tuning speed of the tunable laser and $\theta(t)$ is the phase noise of the LO. For convenience, let $f_{0}$ be the central frequency of the NBF. The impulse response of the NBF in complex form can be written as:

$$
\tilde{h}(t)=h(t) \exp \left(i 2 \pi f_{0} t\right)
$$

where $h(t)$ is the envelope of the impulse response of the NBF. The filtered output at time $t_{0}$ is the convolution between the input signal and the impulse response of the filter:

$$
\tilde{S}\left(t_{0}\right)=\left(\int_{-\infty}^{T}+\int_{T}^{+\infty}\right) \tilde{h}(t) \tilde{S}_{i}\left(t_{0}-t\right) d t
$$

where $T$ is a positive number that is several times larger than the rise time of the NBF, which divides the integral into two sections. The envelope of the impulse response $h(t)$ of a stable and causal linear time-invariant (LTI) system satisfies the following conditions: (a) tends to be 0 when $t$ goes to infinity and (b) equals to 0 when $t<0$. Thus we can approximate the result of (5) by rejecting the integral from $T$ to $+\infty$ and write (5) into (5a) as:

$$
\tilde{S}\left(t_{0}\right) \simeq \int_{0}^{T} \tilde{h}(t) \tilde{S}_{i}\left(t_{0}-t\right) d t
$$

The effect of truncating the integral limit from (5) to (5a) is analyzed in Section III-D. Substituting (3) and (4) into (5a), we obtained the envelope of the signal filtered by the NBF:

$$
S\left(t_{0}\right)=S_{0}\left|\int_{0}^{T} h(t) \exp \left[i 2 \pi v\left(t_{0}-t\right)^{2} / 2+i \theta\left(t_{0}-t\right)\right] d t\right|
$$

For white Gaussian phase noise, the reversal of time integration direction does not change the statistics of $\theta(t)$, therefore we can replace $\theta\left(t_{0}-t\right)$ by $\theta\left(t_{0}+t\right)$ and write (6) into (6a) as:

$$
S\left(t_{0}\right)=S_{0}\left|\int_{0}^{T} h(t) \exp \left[i 2 \pi v\left(t_{0}-t\right)^{2} / 2+i \theta\left(t+t_{0}\right)\right] d t\right|
$$

The phase term in (6a) is composed of two parts. The first phase term is the quadratic phase change due to linear tuning of the LUT. The second phase term is the phase noise from the LO. It can be seen from (6a) that the calculated envelope at given time $t_{0}$ is only determined by the value of the integrands in the time interval from 0 to $T$. For Gaussian noise, the phase noise term $\theta\left(t+t_{0}\right)$ undergoes the same random process as $\theta(t)$ in this time interval. Thus, when we consider the statistical characteristics of the envelope distribution at given frequency position, (6a) can be reduced to:

$$
S\left(t_{0}\right)=S_{0}\left|\int_{0}^{T} h(t) \exp \left[i 2 \pi v\left(t_{0}-t\right)^{2} / 2\right] \exp [i \theta(t)] d t\right|
$$

However, when considering the envelope evolution, (6a) should be used, since there exists the same integration part between the two adjacent times. In the following sections, the parameters in (6a) and (7) are discussed.

\section{A. Phase noise of the $L O$}

For a free-running optical frequency comb, only intracavity noises are present, since there is no phase-lock-loop for stabilizing the comb lines. The fundamental origin of phase noise is quantum noise that arises from amplified spontaneous emission of the gain medium [11]. For this type of noise, the frequency fluctuation $f(t)=d \theta(t) / d t$ is a white Gaussian noise process $f(t) \sim N\left(0,2 \pi \Delta \nu^{2}\right)$, where $\Delta \nu$ is the $3 \mathrm{~dB}$ linewidth of the LO. We can simulate the evolution of the phase noise $\theta(t)$ by integrating independent Gaussian noise, which results in a Brownian motion of the phase:

$$
\theta(t)=\sqrt{2 \pi \Delta \nu} \psi(t)
$$

where $\psi(t)$ is a normalized Brownian motion process with zero-mean and variance $E\left[\psi^{2}(t)\right]=t$.

\section{B. Narrow bandpass filter}

In the frequency calibration systems described in Section II, the NBF acts as a frequency discriminator. For a correct frequency-intensity conversion without distortion, the bandwidth of the NBF should be chosen such that its rise time is smaller than the time required for the chirping frequency to pass through the bandwidth of the NBF. This rule of thumb for bandwidth selection of the NBF was adopted in [3], [4] and more details of the NBF design consideration can be found in [12]. Without considering the phase noise of the LO, the characteristics of the envelope of the calibration signal are related to the type of the NBF and the normalized chirp rate, which is defined as:

$$
\alpha=\frac{v}{B^{2}}
$$




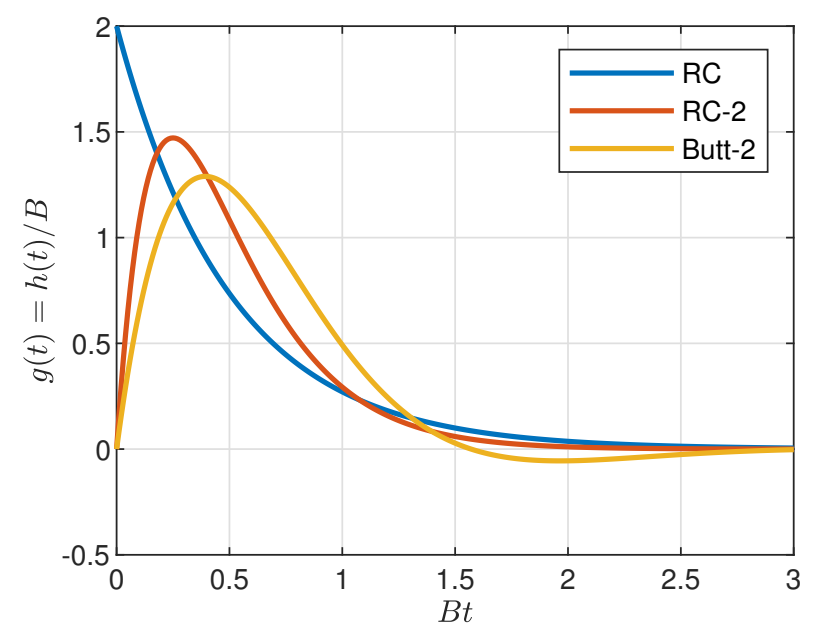

Fig. 2. Envelope of the impulse response of the NBF used in the present work. The functions of the envelope of the impulse response $h(t)$ are $2 B \exp (-2 B t), 16 B[B t \exp (-2 B t)]$ and $4 B \exp (-2 B t) \sin (2 B t)$ for RC, RC-2 and Butt-2 filters respectively. $B$ is the noise-equivalent bandwidth.

where $B$ is the bandwidth of the NBF in Hertz and $v$ is the chirp rate of the heterodyne signal (it is also the tuning speed of the tunable laser) in Hertz per second. It is pointed out by the authors in [12] that the bandwidth selection is a tradeoff between the width of the calibration signal and the large intensity attenuation of its amplitude. Too large normalized tuning speed causes large attenuation and distortion of the calibration signal. In the present work, we limit the normalized tuning speed to $\alpha \leq 0.5$ in order to conform the correct frequency-intensity transform condition.

Three analog filters are used in the simulation: a single tuned filter (RC filter), a two-stage decoupled resonant filter (RC-2 filter) and a second order Butterworth filter (Butt-2 filter). The envelopes of the impulse response of these filters are shown in Fig. 2. For convenience of expressing the impulse response function of the NBF, we use the noise-equivalent bandwidth, which is defined as:

$$
B=\frac{\int_{0}^{+\infty}|h(t)|^{2} d t}{\left|\int_{0}^{+\infty} h(t) d t\right|^{2}}
$$

It can be shown (see Appendix A) that using (10), the envelope of the impulse response functions can be respectively expressed as $2 B \exp (-2 B t), 16 B[B t \exp (-2 B t)]$ and $4 B \exp (-2 B t) \sin (2 B t)$ for $\mathrm{RC}, \mathrm{RC}-2$ and Butt-2 filters respectively, where $B$ is the noise-equivalent bandwidth of the NBFs.

\section{Parameterized expression}

For generality, we normalize the frequency parameters in (6a) and (7), such as the linewidth of the LO, to the bandwidth of the NBF $B$. Meanwhile, we normalize the time parameters, such as the truncation time $T$, to the rise time of the NBF, which is proportional to $1 / B$. By taking $T=\tau / B$ and introducing a normalized time scale $u=t / T$, we rewrite the envelope of filtered calibration signal (6a) in the form that is integrated within unit time interval from 0 to 1 as:

$S_{N}\left(u_{0}\right)=\left|\frac{\tau}{B} \int_{0}^{1} h\left(\frac{u \tau}{B}\right) \exp \left[\frac{i v\left(u_{0}-u\right)^{2} \tau^{2}}{2 B^{2}}\right] \exp \left[i \theta\left(\frac{\tau\left(u+u_{0}\right)}{B}\right)\right] d u\right|$

Here, the amplitude is normalized by taking $S_{N}=S / S_{0}$. Substituting the phase noise expression (8) into (11) and normalizing the linewidth of the LO and the chirp rate by taking $\eta=\Delta \nu / B$ and $\alpha=v / B^{2}$ respectively, we get:

$S_{N}\left(u_{0}\right)=\tau\left|\int_{0}^{1} g(\tau u) \exp \left[\frac{i \alpha\left(u-u_{0}\right)^{2} \tau^{2}}{2}\right] \exp \left[i \sqrt{2 \pi \tau \eta} \psi\left(u+u_{0}\right)\right] d u\right|$

From (11) to (12), we use the property of Brownian motion that $\psi\left(\tau\left(u+u_{0}\right) / B\right)=\sqrt{\tau / B} \psi\left(u+u_{0}\right)$. It is better to use the normalized input instantaneous frequency, which is defined as $\xi=v t / B=\alpha \tau u$, instead of using time as the independent variable:

$S_{N}\left(\xi_{0}\right)=\tau\left|\int_{0}^{1} g(\tau u) \exp \left[\frac{i\left(\xi_{0}-\alpha \tau u\right)^{2}}{2 \alpha}\right] \exp \left[i \sqrt{2 \pi \tau \eta} \psi\left(u+\frac{\xi_{0}}{\alpha \tau}\right)\right] d u\right|$

By this substitution, the envelope of the calibration signal and the amplitude response of the NBF have the same independent variable, facilitating the analysis of the distortion of the signal shape. For example, $\xi=0$ and $\xi=1$ correspond to the time when the heterodyne frequency is equal to the passband central and the bandwidth $B$ of the NBF respectively.

Similarly, the parameterized form of (7) can be written as:

$S_{N}\left(\xi_{0}\right)=\tau\left|\int_{0}^{1} g(\tau u) \exp \left[\frac{i\left(\xi_{0}-\alpha \tau u\right)^{2}}{2 \alpha}\right] \exp [i \sqrt{2 \pi \tau \eta} \psi(u)] d u\right|$

The effect of the phase noise of the LO in calibration of a tunable laser using heterodyne and narrow bandpass filter can now be estimated using (13) and (14). Appendix B summarizes the parameters used in the modeling and simulations.

\section{Error sources in simulation}

Simulations were carried out using numerical integration of (14) to estimate the PDF of the calibration signal envelope at given frequency positions and using (13) for observing the envelope evolution with respect to frequency position. Numerical errors in the simulation are mainly introduced by the following two aspects.

(1). The error induced by discrete time integration. In the simulation, the continuous integration was estimated by numerical integration at times $u_{n}=\{n d\}_{n=1,2, \ldots, N}$, where $d=1 / N$ with $N$ being the number of time moment. (13) and (14) show that for the same time interval $d$, when any of the parameters $\tau, \alpha$ and $\eta$ becomes larger, the numerical estimation deviates more from the continuous time integration. This gives us a method to estimate $d$ by setting $\tau, \alpha$ and $\eta$ at the largest values required and thus see the maximum value of $d$ for which the PDF does not change anymore.

(2). The error induced by truncation of the integration time $\tau$, i.e. the error introduced by using (5a) to estimate (5) similar 


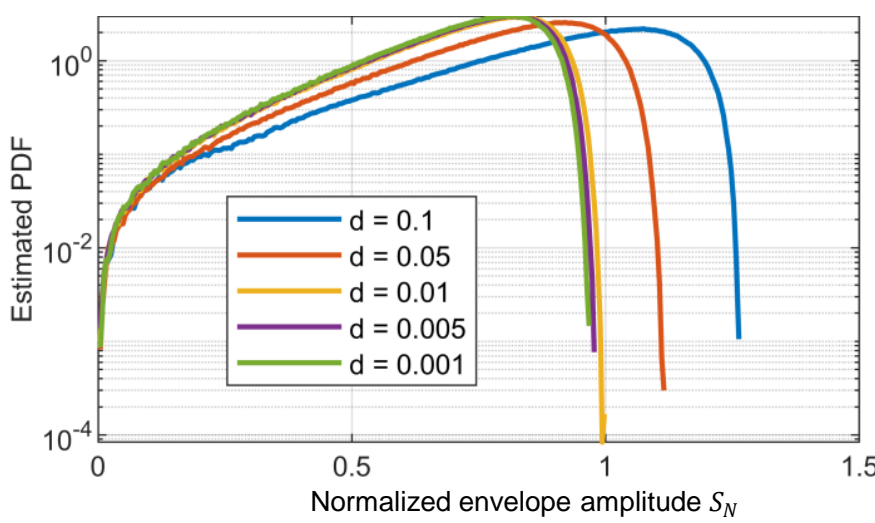

Fig. 3. Effect of sampling time $d$. The estimated PDFs are generated by setting the parameters at the largest value required: $\tau=5, \alpha=0.1$ and $\eta=0.5$.

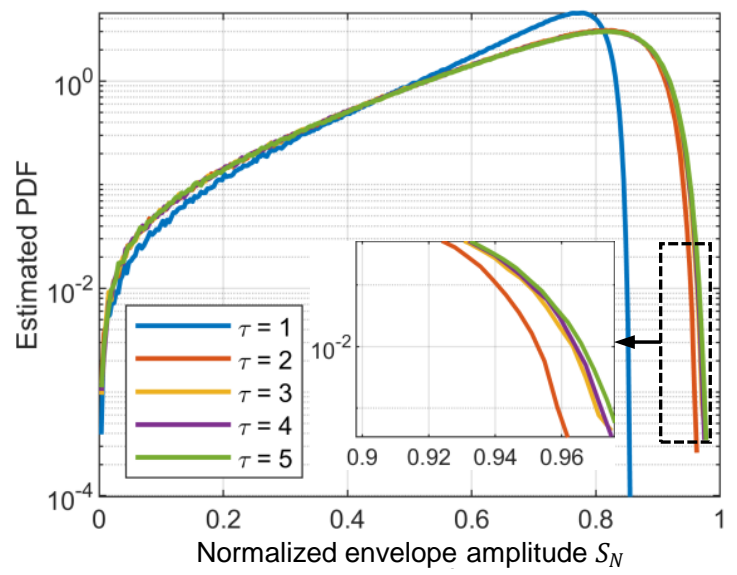

Fig. 4. Effect of integration time truncation. ( $\xi=0, \alpha=0.1$ and $\eta=0.5)$.

to (1). We selected the acceptable value of the truncation time by setting $\tau, \alpha$ and $\eta$ at the largest values and selected a value for which the PDF does not change anymore.

As mentioned in Section III-B, the bandwidth of the NBF should be selected such that the normalized speed is small. Therefore in the present work $\alpha \leq 0.5$ is considered. This indicates that, for a typical laser tuning speed of $10 \mathrm{~nm} / \mathrm{s}$ $(1.25 \mathrm{THz} / \mathrm{s}$ at $1550 \mathrm{~nm})$, the bandwidth should not be smaller than 1.6 MHz. In the present work, we consider the noise level $\eta \leq 0.5$, since the linewidth of a free-running fiberbased frequency comb is reported from tens to hundreds of kilohertz [13]. Taking $\tau=5, \alpha=0.5$ and $\eta=0.5$, the estimated PDF of the envelope amplitude $S_{N}$ with different sampling time $d$ is shown in Fig. 3, in which an RC filter is used. We can see that when $d<0.01$, the error induced by numerical integration becomes neglectable. The following simulations results are based on $d=0.005$.

Fig. 4 shows the results for different truncation times $\tau$, with parameters $\xi=0, \alpha=0.1, \eta=0.5$ and $d=0.005$. We can see that when $\tau>2$, the error induced by the time truncation can be neglected. In the following simulation results, $\tau=5$ is adopted.

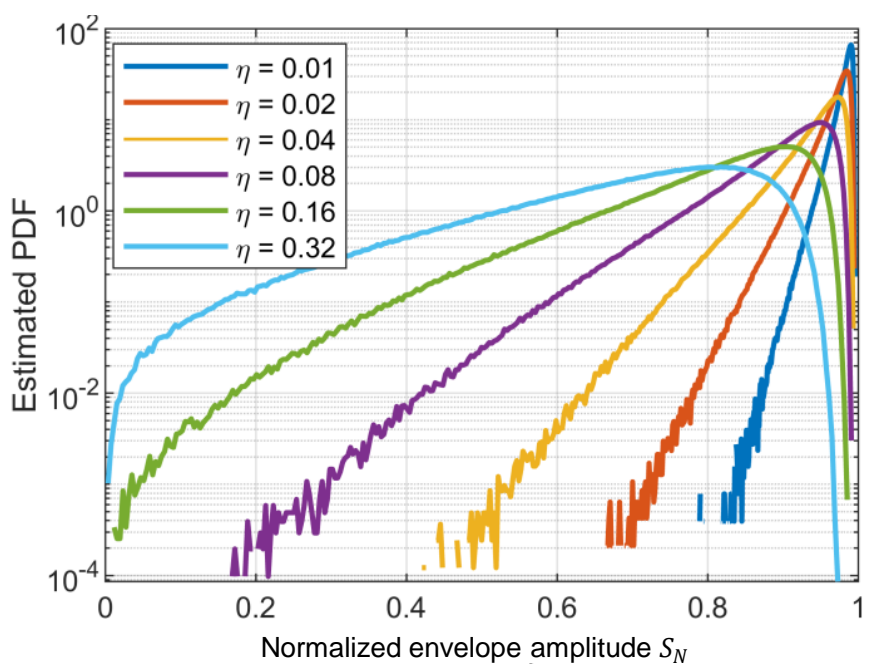

Fig. 5. Estimated PDF of envelope amplitude $S_{N}$ at the passband central frequency $(\xi=0) . \tau=5, \alpha=0.1$.

\section{RESULTS AND DISCUSSION}

\section{A. Probability density function of the envelope amplitude $S_{N}$}

Fig. 5 shows the estimated PDF of the envelope amplitude of the calibration signal with different phase noise levels at the passband center $(\xi=0)$. The parameters were set as $\tau=5$, $\alpha=0.1$ and an RC filter was used. When the phase noise $\eta$ gets larger, the distribution spreads into wider range, becoming more like uniform distribution.

Fig. 6 shows the estimated PDF at different frequency positions $(\xi)$. From the center to the passband edge frequencies, the envelope amplitude that has the maximum probability is decreasing. This is in accordance with the characteristic of the transfer function of the NBF. However, the amplitude distribution is more uniformly distributed at the passband edge frequency. Comparing Fig. 6(a), (b) and (c), we can see that at smaller chirp rate $\alpha$, the distributions at symmetric frequency position of the passband, e.g. $\xi=2$ and $\xi=-2$, are the same (Fig. 6(a)). When the chirp rate $\alpha$ becomes larger, the distribution becomes more asymmetric (Fig. 6(c)).

The distributions using different filters are shown in Fig. 7. A small improvement can be seen between the RC-2 filter and RC filter. Better results can be obtained using Butt-2 filter with values larger than 1 .

\section{B. Distribution of center of mass of the envelope amplitude}

A typical envelope of the calibration signal is shown in Fig. 8 , showing the amplitude fluctuations. The statistical properties of the amplitude at different normalized frequencies have been shown in the previous section. In this section we characterize the overall property of the filtered envelope. The centroid of the envelope is used to determine the instantaneous frequency of the LUT in [3]. Here we define the centroid as the center of mass of the filtered calibration peak, as defined in (2).

The distribution of the center of mass of the envelope is shown in Fig. 9. It can be seen that both the chirp rate and the phase noise affect the uncertainty of the center of mass. Comparing Fig. 9(a) and (b), we see that at small chirp rates, 

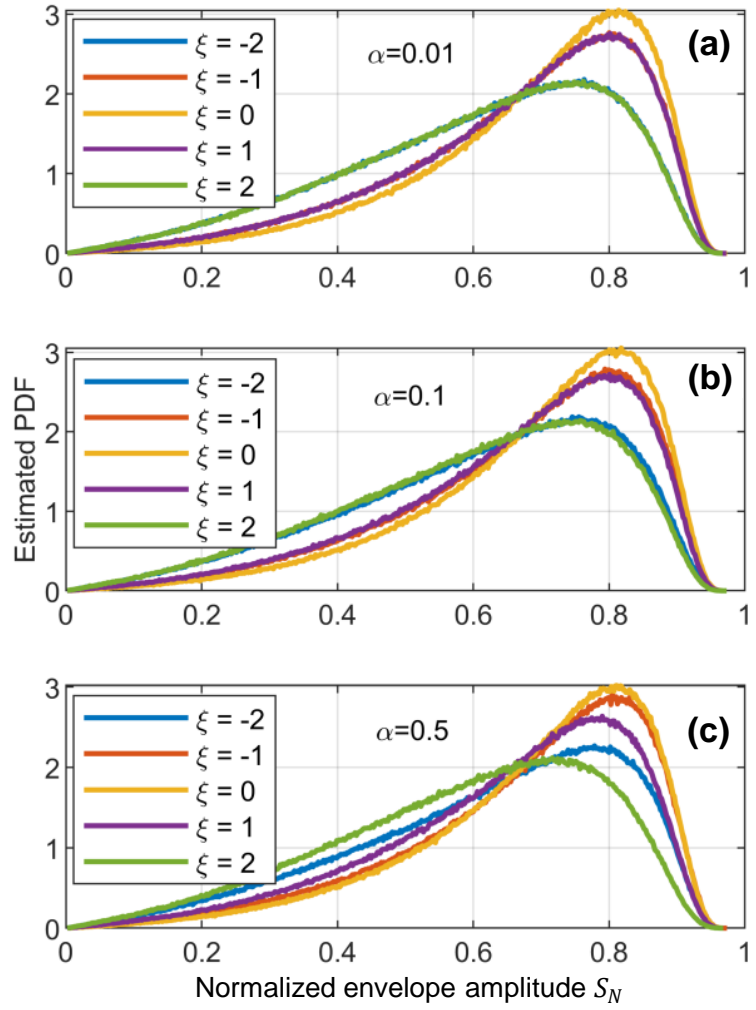

Fig. 6. Estimated PDF at different frequency positions with different chirp rates. (a) $\alpha=0.01$. (b) $\alpha=0.1$. (c) $\alpha=0.5$. Parameters used are $\tau=5$, $\eta=0.32$ with $\mathrm{RC}$ filter.
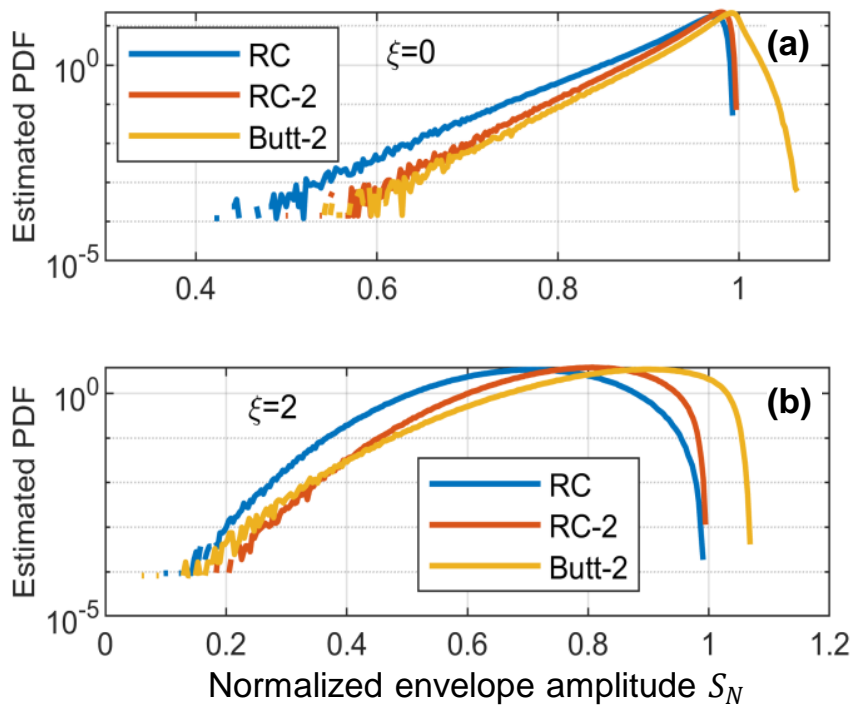

Fig. 7. Estimated PDF with different filters. (a). In frequency position $\xi=0$. (b). In frequency position $\xi=2$. Parameters used are $\tau=5, \alpha=0.1$ and $\eta=0.04$.

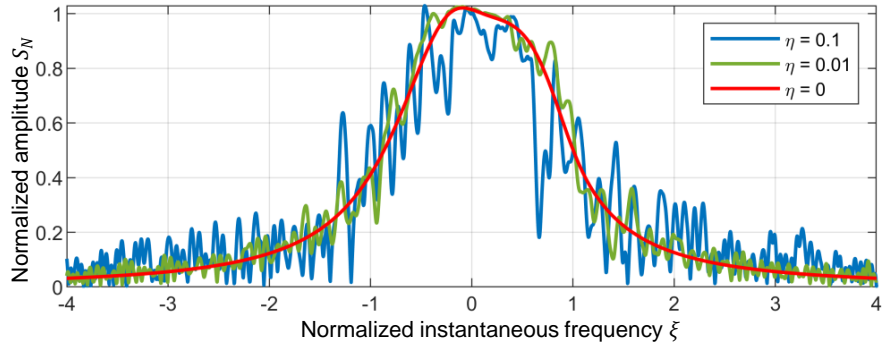

Fig. 8. The envelope of the filtered calibration signal with different phase noise level. Parameters used are $\tau=5, \alpha=0.5$ with RC-2 filter.
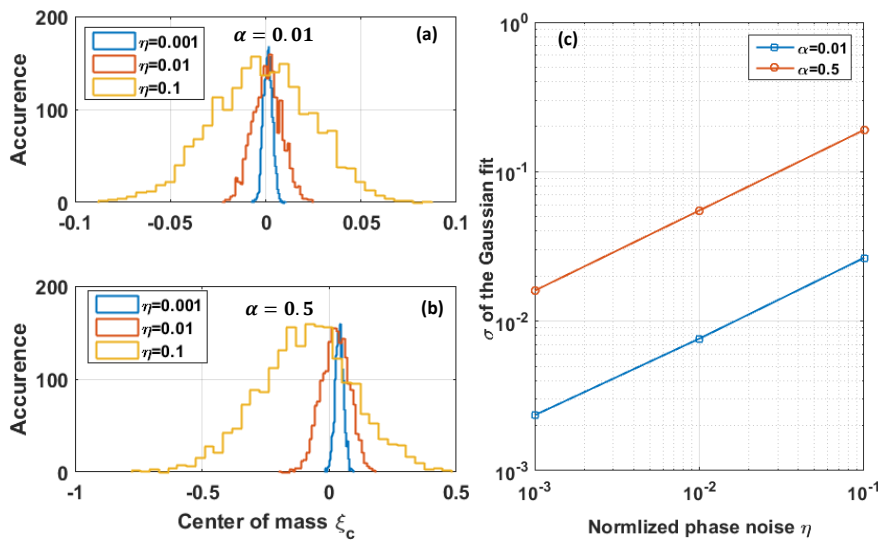

Fig. 9. Distribution of the center of mass of filtered calibration signal with different chirp rates. (a) and (b), histograms of simulation result with $\alpha=$ 0.01 and $\alpha=0.5$ respectively using $\tau=5$ and RC-2 filter. (c), the standard deviation of the Gaussian fit of (a) and (b).

the mean value of the center of mass is at the central frequency of the $\operatorname{NBF}(\xi=0)$, while the mean value deviates from the central frequency of the filter at large chirp rates, even when the phase noise is small. This result is in accordance with the analysis in [12], that the deviation of maximum response under dynamic condition is proportional to the normalized chirp rate.

Using normal distribution to fit the histogram in Fig. 9(a) and (b), the standard deviation of the Gaussian fit of the distribution is shown in Fig. 9(c). We find that the standard deviation of the distribution has a power relationship with the normalized phase noise $\eta$. For $\alpha=0.01$ it gives $\sigma=0.088 \times \eta^{0.53}$ and for $\alpha=0.5$ it gives $\sigma=0.065 \times \eta^{0.54}$.

\section{CONClusion}

Calibrating the instantaneous frequency of a tuning laser is important in many fields, such as tunable diode laser absorption spectroscopy (TDLAS), frequency scanning interferometry (FSI), etc. The developing method using frequency comb as local oscillator in heterodyning and filtering shows high spectral resolution and wide calibration range. For a nonfully stabilized frequency comb, the phase noise of the comb lines affects the calibration signal envelope. In this paper, a model for characterizing the envelope amplitude affected by the Gaussian phase noise of the comb lines is presented. In this model, the tuning speed of the tunable laser, the phase noise level of the LO and the type of the narrow passband filter are taken into account. As shown in the model, the effect of 
these parameters are closely related to the bandwidth of the $\mathrm{NBF}$, that can be expressed in normalized form. It shows that the PDF of the envelope amplitude tends to be a uniformly distributed when the phase noise is larger. At low tuning speed, the PDF distributions has little difference at symmetric frequency positions of the passband of the NBF. When tuning speed increase, these distributions become different. It shows that both the tuning speed and the phase noise level of the LO laser contribute to the center of mass distributions. For example, Gaussian fit of the center of mass distribution shows the standard deviation of $\sigma=0.016$ and $\sigma=0.19$ for the normalized noise level $\eta=10^{-3}$ and $\eta=10^{-1}$ respectively at normalized tuning speed $\alpha=0.5$ for RC filter. Considering the use of an RC filter with noise-equivalent bandwidth $B=1.58$ $\mathrm{MHz}$ in the calibration of a tuning laser with tuning speed $10 \mathrm{~nm} / \mathrm{s}$ at $1550 \mathrm{~nm}(\alpha=0.5)$, the standard deviation of the center of mass is $300 \mathrm{kHz}$ for linewidth $\Delta \nu=158 \mathrm{kHz}$ $(\eta=0.1)$ comb linewidth.

Although the analyses described above are based on analog filters, it is easy to be applied in digital filtering, especially for finite impulse response (FIR) filters, the impulse response of which are finite. This means that there is no truncation error if we take the whole impulse response as the analysis interval in (5) and (5a). For digital infinite impulse response (IIR) filters, the impulse response of the filter is truncated similarly to the analog filters.

\section{APPENDIX A}

\section{IMPULSE RESPONSE OF FILTERS}

In the following, the impulse response function of the NBF expressed in the noise-equivalent bandwidth is derived. The system function of the single tuned RC filter is:

$$
H_{R C}(s)=\frac{\pi B_{c}}{\left(s-i 2 \pi f_{0}\right)+\pi B_{c}}
$$

Where $B_{c}$ and $f_{0}$ are is the $-3 \mathrm{~dB}$ bandwidth and the central frequency of the NBF. By inverse Laplace transform, the impulse response function is:

$$
\tilde{h}_{R C}(t)=\pi B_{c} \exp \left[-\left(\pi B_{c}-i 2 \pi f_{0}\right) t\right]
$$

Using (10), the noise-equivalent bandwidth of the RC filter $B$ can be calculated to be $\pi B_{c} / 2$. Substituting into (A2), the envelope of the impulse response function can be obtained as:

$$
h_{R C}(t)=2 B \exp (-2 B t)
$$

The impulse response function of the RC-2 and Butt-2 filters can be obtained in the similar way.

\section{APPENDIX B}

\section{PARAMETERS SUMMARY}

The parameters used in the modeling and simulations are summarized in Table I.

\section{ACKNOWLEDGMENT}

Yu wants to thank China Scholarship Council (CSC) for the financial support of this work.

\begin{tabular}{|c|c|c|c|}
\hline Parameter & Description & Normalization & Unit \\
\hline$\alpha$ & $\begin{array}{l}\text { Normalized chirp rate of } \\
\text { the heterodyne signal (lin- } \\
\text { ear tuning speed of the } \\
\text { tuning laser). }\end{array}$ & $v / B^{2}$ & - \\
\hline$B$ & $\begin{array}{l}\text { Noise equivalent band- } \\
\text { width of the NBF. }\end{array}$ & - & $\mathrm{Hz}$ \\
\hline$d$ & $\begin{array}{l}\text { The time step in the nu- } \\
\text { merical integration. }\end{array}$ & $1 / \mathrm{N}$ & - \\
\hline$\Delta \nu$ & The linewidth of the LO. & - & $\mathrm{Hz}$ \\
\hline$\eta$ & $\begin{array}{l}\text { Phase noise level. Nor- } \\
\text { malized linewidth of the } \\
\text { LO. }\end{array}$ & $\Delta \nu / B$ & - \\
\hline$h(t)$ & $\begin{array}{l}\text { The impulse response of } \\
\text { the NBF. }\end{array}$ & - & - \\
\hline$v$ & $\begin{array}{l}\text { The tuning speed of the } \\
\text { tunable laser and the chirp } \\
\text { rate of the heterodyne sig- } \\
\text { nal. }\end{array}$ & - & $\mathrm{Hz} / \mathrm{s}$ \\
\hline$\psi(t)$ & $\begin{array}{l}\text { Standard Brownian mo- } \\
\text { tion process. }\end{array}$ & $\theta(t) / \sqrt{2 \pi \Delta \nu}$ & - \\
\hline$S_{N}$ & $\begin{array}{l}\text { Envelope amplitude of the } \\
\text { filtered signal. }\end{array}$ & - & - \\
\hline$T$ & Truncation time. & - & $\mathrm{s}$ \\
\hline$\tau$ & $\begin{array}{l}\text { Normalized truncation } \\
\text { time. }\end{array}$ & BT & - \\
\hline$\theta(t)$ & Phase noise of the LO. & - & $\mathrm{rad}$ \\
\hline$u$ & Normalized time scale. & $t / T=B t / \tau$ & - \\
\hline$\xi$ & $\begin{array}{l}\text { Normalized frequency of } \\
\text { the NBF. }\end{array}$ & $v t / B=\alpha \tau u$ & - \\
\hline
\end{tabular}

TABLE I

PARAMETERS USED IN MODELING AND SIMULATIONS.

\section{REFERENCES}

[1] E. Baumann, F. R. Giorgetta, I. Coddington, L. C. Sinclair, K. Knabe, W. C. Swann, and N. R. Newbury, "Comb-calibrated frequencymodulated continuous-wave ladar for absolute distance measurements," Opt. Lett., vol. 38, no. 12, pp. 2026-2028, Jun 2013.

[2] J. Dale, B. Hughes, A. J. Lancaster, A. J. Lewis, A. J. Reichold, and M. S. Warden, "Multi-channel absolute distance measurement system with sub ppm-accuracy and $20 \mathrm{~m}$ range using frequency scanning interferometry and gas absorption cells," Opt. Express, vol. 22, no. 20 pp. 24 869-24 893, 2014.

[3] J. Jennings, S. Halverson, R. Terrien, S. Mahadevan, G. Ycas, and S. Diddams, "Frequency stability characterization of a broadband fiber fabry-pérot interferometer," Opt. Express, vol. 25, no. 14, pp. 15599 15 613, 72017.

[4] P. Del'Haye, O. Arcizet, M. Gorodetsky, R. Holzwarth, and T. Kippenberg, "Frequency comb assisted diode laser spectroscopy for measurement of microcavity dispersion," Nat. Photonics, vol. 3, no. 9, pp. 529-533, 2009.

[5] K. Feng, J. Cui, H. Dang, W. Wu, X. Sun, X. Jiang, and J. Tan, "An optoelectronic equivalent narrowband filter for high resolution optical spectrum analysis," Sensors, vol. 17, no. 2, p. 348, 2017.

[6] S. Sugavanam, S. Fabbri, S. Le, I. Lobach, S. Kablukov, S. Khorev, and D. Churkin, "Real-time high-resolution heterodyne-based measurements of spectral dynamics in fibre lasers," Sci. Reports, vol. 6, no. 1, p. 23152, 2016.

[7] D. Brunner, X. Porte, M. C. Soriano, and I. Fischer, "Real-time frequency dynamics and high-resolution spectra of a semiconductor laser with delayed feedback," Sci. Reports, vol. 2, no. 1, p. 732, 2012.

[8] J. Alnis, A. Matveev, N. Kolachevsky, T. Udem, and T. Hänsch, "Subhertz linewidth diode lasers by stabilization to vibrationally and thermally compensated ultralow-expansion glass Fabry-Pérot cavities," Phys. Rev. A, vol. 77, no. 5, p. 053809, 2008.

[9] T. Liu, N. R. Newbury, and I. Coddington, "Sub-micron absolute distance measurements in sub-millisecond times with dual free-running 
femtosecond er fiber-lasers." Opt. Express, vol. 19, no. 19, pp. 18 501-9, 2011.

[10] A. Mateo and Z. W. Barber, "Precision and accuracy testing of FMCW ladar-based length metrology." Appl. Optics, vol. 54, no. 19, pp. 601924, 2015.

[11] R. Paschotta, A. Schlatter, S. Zeller, H. Telle, and U. Keller, "Optical phase noise and carrier-envelope offset noise of mode-locked lasers," Appl. Phys. B, vol. 82, no. 2, pp. 265-273, 2006.

[12] W. Yu, P. Pfeiffer, and J. Fontaine, "Filter design for dynamic frequency calibration of an external cavity diode tunable laser using frequency comb," Proc. SPIE, vol. 10680, pp. 10680 - 10680 - 8, 2018.

[13] N. R. Newbury and W. C. Swann, "Low-noise fiber-laser frequency combs," J. Opt. Soc. Am. B, vol. 24, no. 8, pp. 1756-1770, 2007. 